\title{
Effect of odanacatib on root resorption and alveolar bone metabolism during orthodontic tooth movement
}

\author{
X.X. Wei ${ }^{1,2}$, J.P. Chu ${ }^{3}$, Y.Z. Zou' ${ }^{2}$, N. Ru' ${ }^{1}$, S.X. Cui ${ }^{2}$ and Y.X. Bai ${ }^{1}$ \\ ${ }^{1}$ Department of Orthodontics, Capital Medical University School of Stomatology, \\ Beijing, China \\ ${ }^{2}$ Department of Orthodontics, Zhengzhou University School of Stomatology, \\ Zhengzhou, China \\ ${ }^{3}$ Department of Endodontics, Zhengzhou University School of Stomatology, \\ Zhengzhou, China \\ Corresponding author: Y.X. Bai \\ E-mail: yuxingb@163.com
}

Genet. Mol. Res. 14 (4): 17972-17981 (2015)

Received September 8, 2015

Accepted November 18, 2015

Published December 22, 2015

DOI http://dx.doi.org/10.4238/2015.December.22.23

ABSTRACT. The aim of this study was to investigate the effect of local administration of odanacatib (ODN) on orthodontic root resorption and the status of alveolar bone metabolism in rat molars. All specimens were scanned using microcomputed tomography and then the raw images were reconstructed. The total volume of the root resorption craters of the 60 $\mathrm{g}-\mathrm{NS}$ (normal saline) group was higher than in the $60 \mathrm{~g}$-ODN group and the control group. In the $60 \mathrm{~g}$-NS group, the bone volume fraction values of alveolar bone were significantly decreased compared with the other 2 groups. There were no significant differences in the bone volume fraction values of the tibiae among the 3 groups. The results of tartrate-resistant acid phosphatase-positive (TRAP+) numbers showed that there was no difference between the $60 \mathrm{~g}$-NS group and the $60 \mathrm{~g}$-ODN group. The expression of cathepsin $\mathrm{K}$ was decreased significantly in the $60 \mathrm{~g}$-ODN group. These results indicate that ODN reduces orthodontics-induced 
external root resorption and increases alveolar bone metabolism. This may be because ODN inhibits the activity of odontoclasts, but maintains the quantity of odontoclasts and enhances bone formation. ODN promotes local alveolar bone metabolism, but does not affect systemic bone metabolism.

Key words: Microcomputed tomography; Odanacatib; Root resorption

\section{INTRODUCTION}

Although orthodontics-induced external root resorption (OERR) may be partially repaired by cementum, severe OERR ( $>5 \mathrm{~mm}$ ) can destroy the root structure and lead to tooth loss. Given the harmful effect of OERR on dental health, numerous studies have examined how to reduce or eliminate root resorption. The morphology and function of the cells in both root resorption and bone resorption show many similarities, and thus the process of root resorption is similar to that of bone resorption.

Bone undergoes continuous resorption by osteoclasts and replacement by osteoblasts. When resorption exceeds formation, osteoporosis occurs. Currently, antiresorptive therapy for osteoporosis prevents further bone loss but also inhibits bone formation, and thus does not cure osteoporosis. New methods are needed to overcome this problem. Drake et al. (1996) reported that osteoclasts were mainly responsible for bone degradation during bone resorption. Additionally, cathepsin $\mathrm{K}(\mathrm{CK})$ is a lysosomal cysteine protease that is highly expressed in osteoclasts, but expressed at extremely low levels in other cells (Drake et al., 1996). Thus, CK may play a key role in mediating bone resorption.

CK knockout mice exhibit show increased bone mineral density (BMD), thickened bone trabeculae, and increased bone strength compared with wild-type mice (Pennypacker et al., 2009). In addition, reduced bone resorption and increased BMD after selective inhibition of CK have been demonstrated in both preclinical and clinical studies. Thus, CK should be inhibited to reduce or eliminate bone resorption. Interestingly, Gauthier et al. (2008) reported that odanacatib (ODN) was a potent and selective inhibitor of $\mathrm{CK}$ in in vivo and in vitro models. A clinical study in postmenopausal women demonstrated that ODN significantly increased BMD and reduced biochemical markers of bone resorption (Eisman et al., 2011). Moreover, ODN was shown to prevent the loss of BMD in ovariectomized rabbits (Pennypacker et al., 2011) and rhesus monkeys (Fratzl-Zelman et al., 2013). Histomorphometric analysis in both species has suggested that ODN treatment preserves normal bone biomechanical properties.

Previous studies have demonstrated that ODN is capable of inhibiting the expression of CK in osteoclasts; therefore, ODN can effectively reduce bone resorption. Sasaki et al. (1988) suggested that odontoclastic resorption of the tooth root proceeds in a manner similar to that in osteoclastic bone resorption. In contrast, Gonzales et al. (2009) found that like osteoclasts, odontoclasts could express CK during orthodontic tooth movements. No study has reported the relationship between the local administration of ODN and the expression levels of CK in odontoclasts leading to orthodontic root resorption. Therefore, we investigated the effect of ODN on orthodontic root resorption and bone metabolism and provide a scientific basis for reducing OERR in the orthodontic clinic.

\section{MATERIAL AND METHODS}

\section{Animals}

This study examined 24 8-week-old male Sprague-Dawley rats. These animals were kept 
in plastic cages with access to standardized laboratory rat diet and water ad libitum. All protocols were approved by the Ethics Committee of Beijing Stomatological Hospital.

After the acclimatization period (1 week), the rats were randomly divided into 3 groups. The $60 \mathrm{~g}$-NS (normal saline) group received $60 \mathrm{~g}$ orthodontic force and local NS injection; the 60 $\mathrm{g}$-ODN group received $60 \mathrm{~g}$ orthodontic force and local ODN injection, while the control group was not subjected to orthodontic force. There were 8 rats in each group.

\section{Orthodontic appliance placement and local administration of ODN}

The forced rats were weighed and then injected intraperitoneally with $10 \% 300 \mathrm{mg} / \mathrm{kg}$ chloralic hydras for general anesthesia. A cervical groove was prepared using a round bur with a dental low-speed handpiece on the distal and labial surfaces of 2 incisors just above the gingival margin. The orthodontic nickel-titanium closed coil spring (GRINM, Beijing, China) was ligated between the upper right first molar and both incisors using a $0.20-\mathrm{mm}$ steel ligature. Next, the spring was activated for approximately $1 \mathrm{~mm}$ to produce a continuous force of $60 \mathrm{~g}$ to move the upper right first molar forward (Figure 1). A force of $60 \mathrm{~g}$ was used because the amount of force typically used to move rat molars in the mesial direction is 50-60 g (Gonzales et al., 2009). In order to enhance retention around the teeth, the ligature wire was secured using bond adhesive (Transbond, 3M Unitek, Monrovia, CA, USA) on the incisors. Rats in the $60 \mathrm{~g}$-ODN group were injected with $50 \mathrm{~mL} 1.25 \mathrm{mM}$ ODN (MK0822; Selleckchem, Houston, TX, USA) into the buccal groove around the upper right first molar every third day. Rats in the $60 \mathrm{~g}$-NS group were injected with $50 \mathrm{~mL}$ NS. Spring retention was evaluated daily to ensure the stability of the applied force. The diet of the rats did not change after the orthodontic device had been applied.

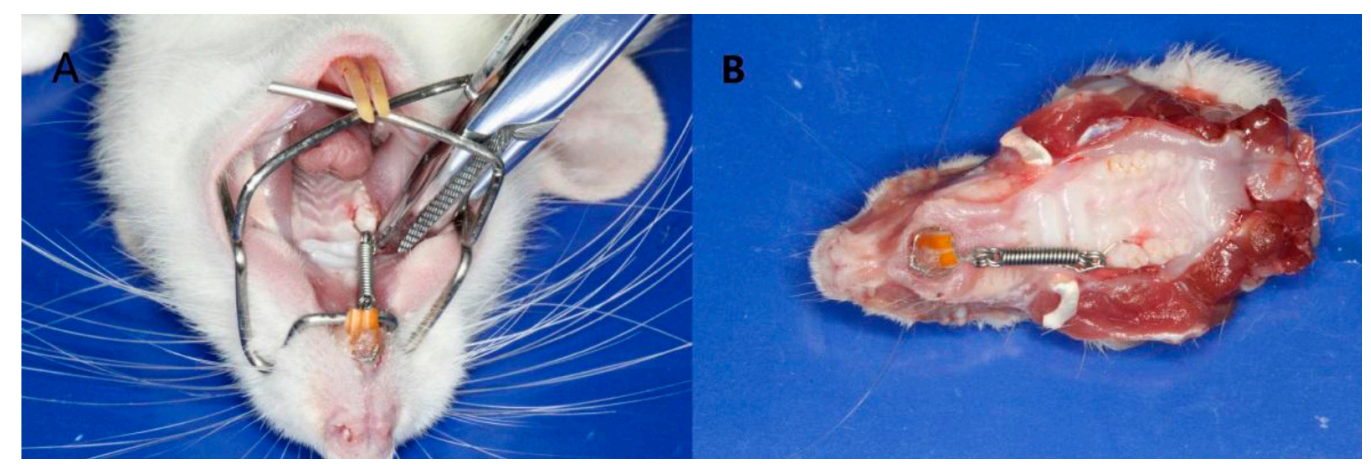

Figure 1. NiTi coil was ligated to the upper right first molar with a ligature.

\section{Microcomputed tomography (micro-CT) scanning and analysis}

After 15 days, the rats were sacrificed and the maxilla and right tibiae of each animal were removed and placed in 10\% formalin for fixation. Specimens were scanned individually using a micro-CT (SkyScan 1076, Kontich, Belgium). The source voltage for the micro-CT for the maxillary images was $70 \mathrm{kV}$ and the source current was $141 \mu \mathrm{A}$, with a rotation step of $0.84^{\circ}$ and an image voxel size of $9.49 \mathrm{~mm}$. The scan duration was approximately $20 \mathrm{~min}$. The source voltage and the source current of the micro-CT for the tibiae images were the same as were used for the maxillary images, with a rotation step of $0.6^{\circ}$ and an image voxel size of $18.97 \mathrm{~mm}$. The scan duration was 
approximately $10 \mathrm{~min}$. Reconstruction of the raw data in 2D transverse slices was performed using NRecon software (Bruker, Kontich, Belgium) (Figure 2).

To analyze all images, individual threshold values were averaged and a representative value was used to threshold these images using CTAn (V.1.14.4, Bruker, Kontich, Belgium). Measurements of bone volume fraction (BVF) in the first molar furcation area were carried out as previously described (Park et al., 2007). The furcation area was chosen because to provide reproducible, morphological landmarks. During BVF measurements of the tibiae, the trabecular compartment was segmented from the image by subtracting the cortical region beneath the growth plate of tibiae, and then the trabecular were established as the "volume of interest" (Figure 3). The $3 \mathrm{D}$ image reconstruction at the appropriate cross-section was carried out using Mimics 17 software (Materialise, Leuven, Belgium), and the mesial root of the upper right first molar was separated manually. The volume of root resorption craters on the root surface was analyzed based on a convex hull algorithm similar to that used by Harris et al. (2006).
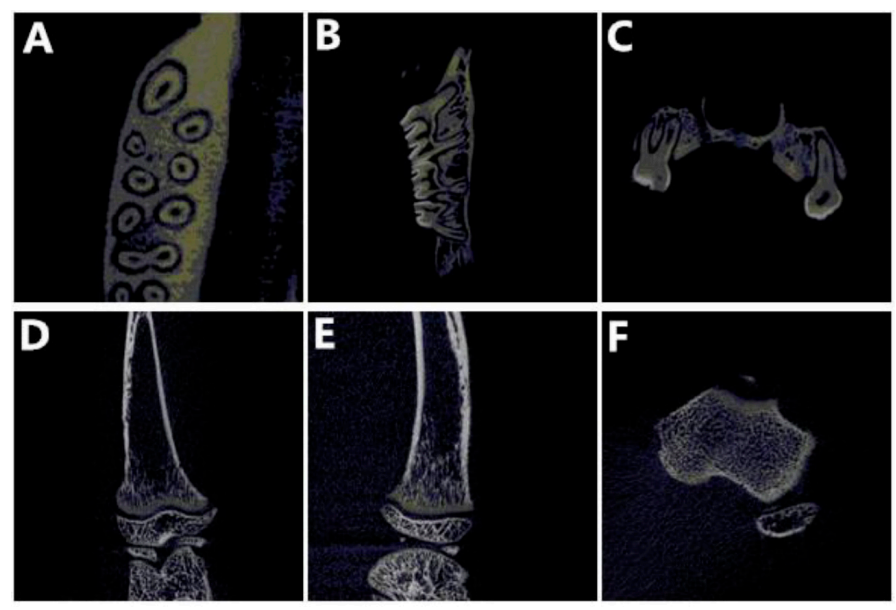

Figure 2. A. B. C. Three different directional slices of the upper right first molar; D. E. F. three different directional slices of the right tibiae.
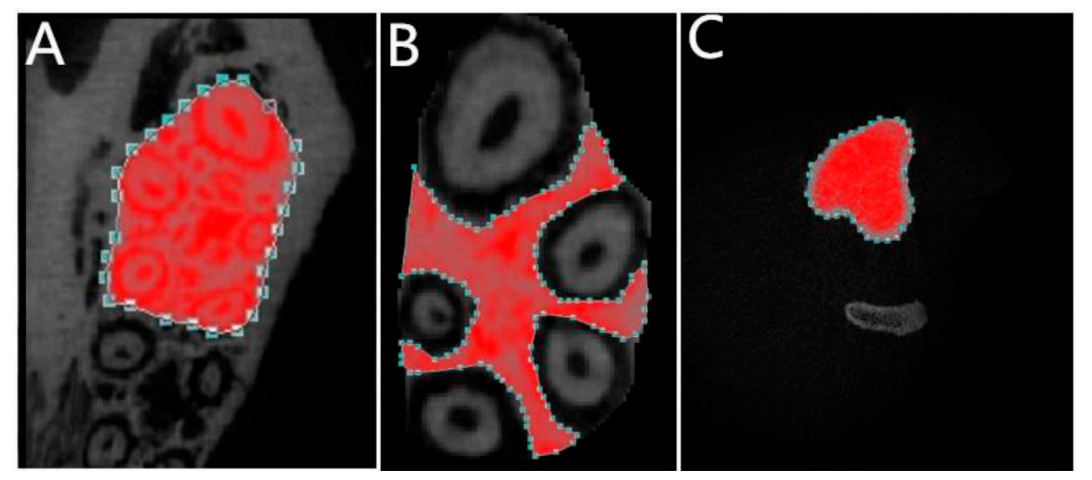

Figure 3. A. and B. Method for creating 3-D ROls used in analysis of alveolar bone; C. method for creating 3-D ROls used in analysis of tibiae. 


\section{Histological and immunohistochemical analysis of CK expression}

After micro-CT scanning, the specimens were decalcified using $10 \%$ ethylene diamine tetraacetic acid for 4 weeks at $4^{\circ} \mathrm{C}$, and then processed for embedding in paraffin. Eight sections per specimen were produced from the mesial to the distal of each upper right first molar. Half of the samples were used to count the tartrate-resistant acid phosphatase-positive (TRAP+) numbers manually on the mesial compression side from pictures acquired using an optical microscope at 400 ' magnification. The other half of the samples was used for immunohistochemical staining to detect CK protein expression. Immunohistochemical staining specimens were deparaffinized and then incubated with anti-CK antibody (Santa Cruz Biotechnology, Santa Cruz, CA, USA) overnight at $4^{\circ} \mathrm{C}$. Next, the slides were stained using the 2-step plus poly-horseradish peroxidase anti-rabbit IgG detection system (ZSGB-Bio, Beijing, China), followed by color development with diaminobenzidine. Integrated optical density was measured to determine the intensity of immunohistochemistry signals using the Biosens Digital Imaging System v1.6 (Bio-tech Co., Ltd., Shanghai, China).

\section{Statistical analysis}

Statistical analysis was performed using SPSS version 19.0 (SPSS Inc., Chicago, IL, USA). One-way analysis of variance was used to determine differences between groups. All data are reported as means \pm standard deviation. Differences for which $P$ values were $<0.05$ were considered to be significant.

\section{RESULTS}

\section{Volume of root resorption craters}

Based on the application of a 3-D convex hull, the total volume of root resorption craters was measured in the 3 groups. There was no significant difference between the control group and the $60 \mathrm{~g}$-ODN group, and the volume of the $60 \mathrm{~g}$-ODN group was higher than that of the control group. However, the volume of the root resorption craters in the $60 \mathrm{~g}-\mathrm{NS}$ group was significantly increased $(P<0.001)$ compared to the other 2 groups (Figure 4$)$.

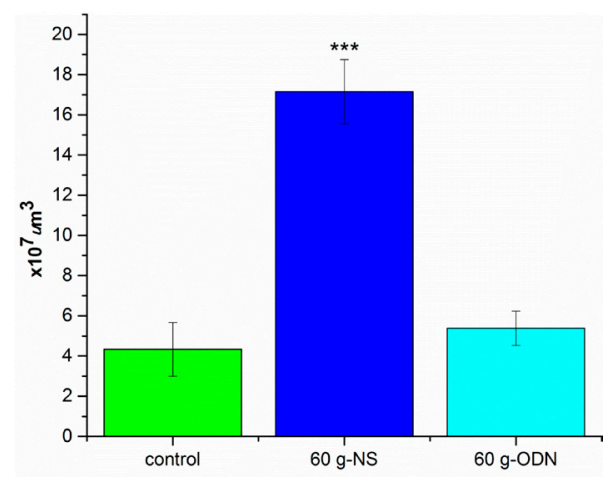

Figure 4. Root resorption crater volume in different groups. Volume of the root resorption craters in the $60 \mathrm{~g}-\mathrm{NS}$ group was significantly increased $(P<0.001)$; there was no significant difference between the control group and the 60 g-ODN group. 


\section{Values of BVF}

To evaluate the effect of ODN on local bone metabolism, the values of BVF were analyzed. In the $60 \mathrm{~g}-\mathrm{NS}$ group, BVF was significantly decreased compared to in the control group and the 60 g-ODN group $(P<0.05)$. However, no significant difference was found between the control group and the $60 \mathrm{~g}$-ODN group (Figure 5). The values for BVF of the tibiae were not significantly different among the 3 groups (Figure 6 ).

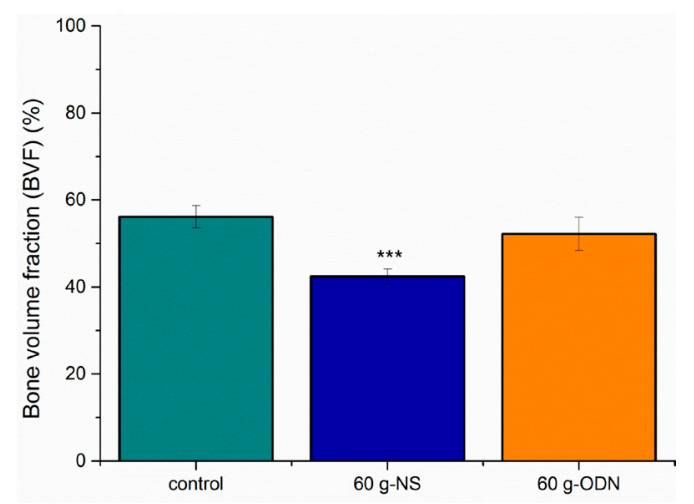

Figure 5. BVF values of alveolar bone in different groups. In the $60 \mathrm{~g}-\mathrm{NS}$ group, BVF was significantly decreased compared to the control group and the $60 \mathrm{~g}-\mathrm{ODN}$ group $(\mathrm{P}<0.001)$.

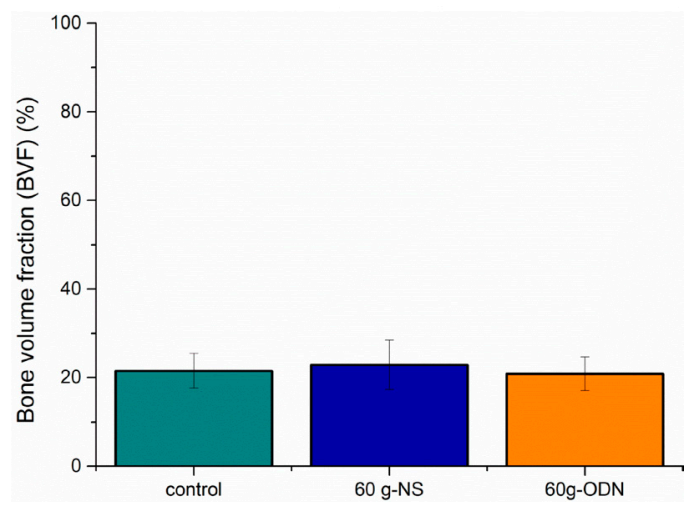

Figure 6. BVF values in tibiae in different groups. There were no significant differences in BVF values among the 3 groups.

\section{TRAP+ numbers and CK expression}

The results of TRAP+ analysis (odontoclast and osteoclast) revealed no difference between the $60 \mathrm{~g}-\mathrm{NS}$ group and the $60 \mathrm{~g}-\mathrm{ODN}$ group. However, these groups showed significant differences compared to the control group $(P<.001)$ (Table 1 and Figure 7$)$. Immunohistochemical analysis showed that the expression of CK decreased significantly in the 60 g-ODN group, which was very similar to that in the control group. In contrast, CK expression levels were very high in the 60 g-NS group $(P<0.001)$ (Figures 8 and 9$)$. 
Table 1. TRAP+ numbers on the compression side of the mesial root of upper left first molar in different groups (means \pm standard deviation).

\begin{tabular}{lc}
\hline Group & TRAP+ numbers \\
\hline Control & $7.70 \pm 1.48^{\mathrm{a}}$ \\
60 g-NS & $30.33 \pm 3.05^{\mathrm{b}}$ \\
60 g-ODN & $29.43 \pm 3.53^{\mathrm{b}}$ \\
\hline
\end{tabular}

a,bIndicate that the value of the 2 groups was significantly different $(P<0.001)$.

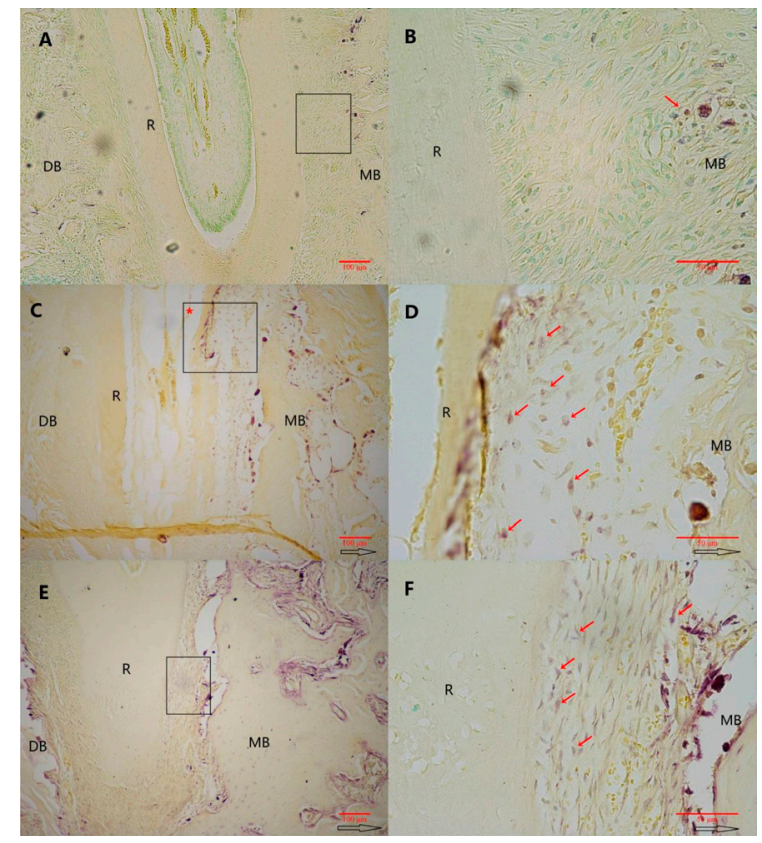

Figure 7. TRAP+ numbers in different groups. A. Control group (without mechanical loading); B. magnified view of the identified area in (A); C. 60 g-NS group; D. magnified view of the identified area in (C); E. 60 g-ODN group; $\mathbf{F}$. magnified view of the identified area in (E); 400X magnification; MB, mesial alveolar bone; DB, distal alveolar bone; R, root; asterisk, root resorption area; red arrows indicate odontoclast and osteoclast; blank arrows indicate the direction of tooth movement.

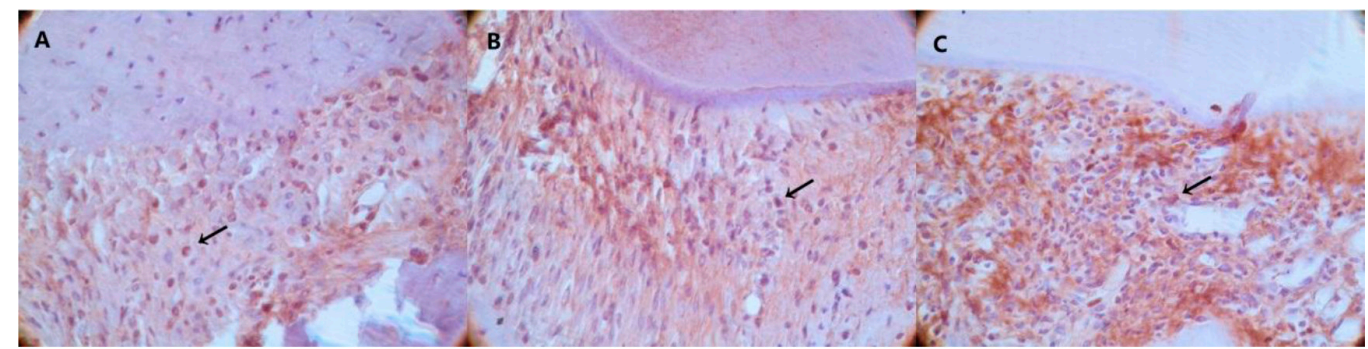

Figure 8. CK expression in different groups. A. Control group; B. 60 g-NS group; C. 60 g-ODN group. Scale $=50 \mu \mathrm{m}$. Black arrow, CK. 


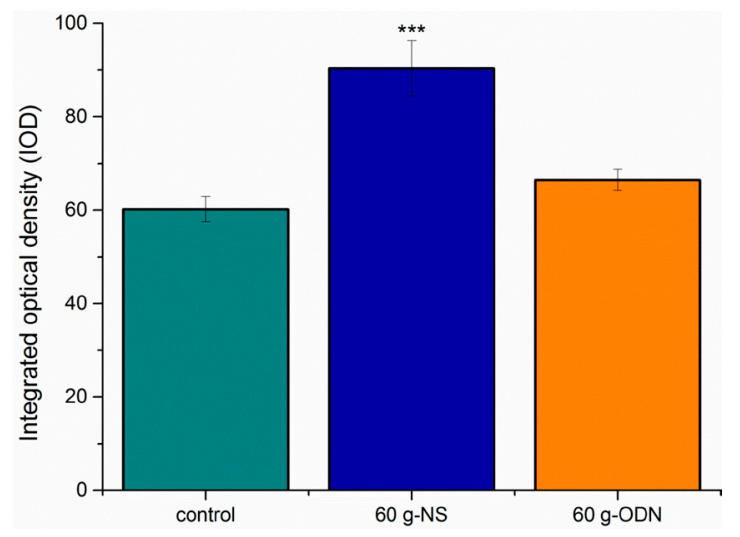

Figure 9. IOD of CK in different groups. The CK expression levels were very high in the $60 \mathrm{~g}-\mathrm{NS}$ group compared to the other two groups $(\mathrm{P}<0.001)$.

\section{DISCUSSION}

Rats are generally considered to be a good model for studying orthodontic tooth movement, which has several practical advantages. First, the number of teeth, classification, periodontal tissue, and anatomical structure are similar to that in humans. Second, they are relatively inexpensive and bred easily, which facilitates the use of large samples. Finally, tissue development during root formation and tissue changes incident to orthodontic treatment appear to be faster in rats than in humans, although their principal mechanisms are the same. Therefore, rats are generally considered to be the best choice for studying experimental orthodontic tooth movement.

Kim et al. (2006) and Lark et al. (2002) reported OST-4077 and SB-331750 as inhibitors of $\mathrm{CK}$ that can effectively prevent bone resorption in ovariectomized rats. However, compared to OST-4077 and SB-331750, ODN has shown excellent pharmacokinetic properties, a longer halflife, and a clear selective effect in clinical studies. The effect of ODN on the microarchitecture of the alveolar bone and OERR has not been examined in previous studies. In this study, the volume of the root resorption craters was not zero in the control group, but was minimal in the 3 groups, even in rats that did not receive orthodontic force. Similar results were observed by Foo et al. (2007). In the $60 \mathrm{~g}$-ODN group, the volume of the root resorption craters was significantly lower than in the $60 \mathrm{~g}$-NS group. There were no differences between the $60 \mathrm{~g}$-ODN group and the control group. These results revealed that ODN could reduce OERR. Since odontoclasts and cementoblasts are responsible for root resorption and repair (Brezniak et al., 2002), the biological mechanism underlying these findings may be that ODN inhibits the resorptive activity of odontoclasts and may promote the differentiation of cementoblasts during orthodontic tooth movement.

In this study, minimal TRAP+ numbers were observed in the control group. However, after the application of $60 \mathrm{~g}$ orthodontic force, the TRAP+ numbers significantly increased. Interestingly, in the $60 \mathrm{~g}$-ODN group, the TRAP+ numbers were similar to those in the $60 \mathrm{~g}-\mathrm{NS}$ group. As root resorption is caused mainly by odontoclasts and osteoclasts and the number of osteoclasts did not change (Gauthier et al., 2008), ODN may help maintain the quantity of odontoclasts.

As described above, the quantity of odontoclasts was found to be normal in this study. ODN decreased OERR and increased alveolar bone metabolism, possibly because the activity of odontoclasts was inhibited. In this study, the results showed that CK expression was significantly 
decreased in the $60 \mathrm{~g}$-ODN group, which was similar to that in the control group. These data suggest that ODN inhibits the expression of $\mathrm{CK}$, which is typically highly expressed in odontoclasts.

Furthermore, it was reported that $\mathrm{CK}$ degrades the organic matrix of bone while allowing demineralization (Atley et al., 2000). Therefore, ODN affects the normal activation of odontoclasts.

Typically, root resorption is measured 15 days after appliance activation to allow for completion of bone remodeling. BVF is an important parameter describing the trabecular microstructure. In addition, micro-CT analysis, a non-destructive examination, can be used to measure BVF with high accuracy and precision (Ding et al., 1999). Therefore, evaluated bone metabolism status based on the value of BVF (Tanck et al., 2001). The results of the present study revealed a statistically significant decrease in BVF values in the $60 \mathrm{~g}-\mathrm{NS}$ group compared with the other 2 groups. In the $60 \mathrm{~g}-\mathrm{ODN}$ group, the values of BVF were similar to that in the control group, indicating that ODN is capable of promoting local alveolar bone metabolism. Surprisingly, recent preliminary reports have suggested that CK-inhibitors prevent the degradation not only of collagen, but also of other proteins, including insulin-like growth factor I and bone morphogenetic protein-2 embedded in the matrix (Fuller et al., 2008). Therefore, we hypothesize that this unique characteristic will help explain the capacity of ODN, as a CK inhibitor, to destroy the function of odontoclasts and enhance alveolar bone formation.

Since ODN is a key regulator of bone remodeling, we examined whether localized ODN affects local and systemic bone metabolism. The micro-CT results showed that ODN affects alveolar bone metabolism, as BVF values in the $60 \mathrm{~g}$-ODN group were significantly increased compared to in the $60 \mathrm{~g}-\mathrm{NS}$ group. However, there were no significant differences in the BVF values for the tibiae among the 3 groups. These data indicate that the ODN inhibits bone resorption only in the alveolar bone, but not in the tibia bone, which was far from the injection site.

Recently, bisphosphonates have been the most commonly prescribed medication for OERR; however, this treatment can increase the possibility of localized jaw osteonecrosis, particularly in postmenopausal patients on long-term bisphosphonate therapy (Ghoneima et al., 2009). According to previous studies, bisphosphonates not only halts bone loss but also inhibits bone formation, causing major therapeutic difficulties. In our study, ODN was administrated to investigate its effect on OERR. Its advantages compared to the commonly root resorption inhibitor were demonstrated, including reduced root resorption and enhanced bone formation.

\section{Conflicts of interest}

The authors declare no conflict of interest.

\section{ACKNOWLEDGMENTS}

Research supported by grants from the Science \& Technology Foundation of Henan Province (\#142102310332), the Technology Bureau of Zhengzhou (\#14PPTGG452), and the National Natural Science Foundation of China (\#81141119).

\section{REFERENCES}

Atley LM, Motr JS, Lalumiere M and Eyre DR (2000). Proteolysis of human bone collagen by cathepsin K: characterization of the cleavage sites generating by cross-ilnked N-telopeptide neoepitope. Bone 26: 241-247.

Brezniak N and Wasserstein A (2002). Orthodontically induced inflammatory root resorption. Part I: the basic science aspects. Angle Orthod. 72: 175-179. 
Ding M, Odgaard A and Hvid I (1999). Accuracy of cancellous bone volume fraction measured by micro-CT scanning. J. Biomech. 32: 323-326.

Drake FH, Dodds RA, James IE, Connor JR, et al. (1996). Cathepsin K, but not cathepsins B, L, or S, is abundantly expressed in human osteoclasts. J. Biol. Chem. 21: 12511-12516.

Eisman JA, Bone HG, Hosking DJ, McClung MR, et al. (2011). Odanacatib in the treatment of postmenopausal women with low bone mineral density: three-year continued therapy and resolution of effect. J. Bone Miner. Res. 26: 242-251.

Foo M, Jones A and Darendeliler MA (2007). Physical properties of root cementum: Part 9. Effect of systemic fluoride intake on root resorption in rats. Am. J. Orthod. Dentofacial Orthop. 131: 34-43.

Fratzl-Zelman N, Roschger P, Fisher JE, Duong le T, et al. (2013). Effects of odanacatib on bone mineralization density distribution in thoracic spine and femora of ovariectomized adult rhesus monkeys: a quantitative backscattered electron imaging study. Calcif Tissue Int. 92: 261-269.

Fuller K, Lawrence KM, Ross JL, Grabowska UB, et al. (2008). Cathepsin K inhibitors prevent matrix-derived growth factor degradation by human osteoclasts. Bone 42: 200-211.

Gauthier JY, Chauret N, Cromlish W, Desmarais S, et al. (2008). The discovery of odanacatib (MK-0822), a selective inhibitor of cathepsin K. Bioorg. Med. Chem. Lett. 18: 923-928.

Ghoneima AA, Allam ES, Zunt SL and Windsor LJ (2009). Bisphosphonates treatment and orthodontic considerations. Orthod. Craniofac. Res. 13: 1-10.

Gonzales C, Hotokezaka H, Matsuo K, Shibazaki T, et al. (2009). Effects of steroidal and nonsteroidal drugs on tooth movement and root resorption in the rat molar. Angle Orthod. 79: 715-726.

Harris DA, Jones AS and Darendeliler MA (2006). Physical properties of root cementum: part 8. Volumetric analysis of root resorption craters after application of controlled intrusive light and heavy orthodontic forces: a microcomputed tomography scan study. Am. J. Orthod. Dentofacial Orthop. 130: 639-647.

Kim MK, Kim HD, Park JH, Lim Jl, et al. (2006). An orally active cathepsin K inhibitor, furan-2-carboxylic acid, 1-\{1-[4-fluoro-2(2-oxo-pyrrolidin-1-yl)-phenyl]-3-oxopiperidin-4-ylcarbamoyl-cyclohexyl)-amide (OST-4077), inhibits osteoclast activity in vitro and bone loss in ovariectomized rats. J. Pharmacol. Exp. Ther. 318: 555-562.

Lark MW, Stroup GB, James IE, Dodds RA, et al. (2002). A potent small molecule, nonpeptide inhibitor of cathepsin K (SB331750 ) prevents bone matrix resorption in the ovariectomized rat. Bone 30: 746-753.

Park CH, Abramson ZR, Taba M Jr, Jin Q, et al. (2007). Three-dimensional micro-computed tomographic imaging of alveolar bone in experimental bone loss or repair. J. Periodontol. 78: 273-281.

Pennypacker B, Shea M, Liu Q, Masarachia P, et al. (2009). Bone density, strength, and formation in adult cathepsin K (-/-) mice. Bone 44: 199-207.

Pennypacker BL, Duong le T, Cusick TE, Mararachia PJ, et al. (2011). Cathepsin K inhibitors prevent bone loss in estrogendeficient rabbits. J. Bone Miner. Res. 26: 252-262.

Sasaki T, Motegi N, Suzuki H, Watanabe C, et al. (1988). Dentin resorption mediated by odontoclasts in physiological root resorption of human deciduous teeth. Am. J. Anat. 183: 303-315.

Tanck E, Homminga J, van Lenthe GH and Huiskes R (2001). Increase in Bone Volume Fraction Precedes Architectural Adaptation in Growing Bone. Bone 28: 650-654. 